\title{
Assessment of Psychophysiological Differences in Eating Disorders after an Integrated Treatment
}

\author{
Pruneti C. ", Cosentino C., Agostinelli F., Sacco M., Innocenti A \\ Department of Clinical and Experimental Medicine, Clinical Psychology Unit, University of Parma, Italy
}

Copyright (C) 2015 by authors, all rights reserved. Authors agree that this article remains permanently open access under the terms of the Creative Commons Attribution License 4.0 International License

\begin{abstract}
The objective of this study was to assess the effectiveness of integrated therapy (IT) in a group of patients with Eating Disorders (EDs; $N=19$ ), divided into $A N$ (Anorexia Nervosa) and BN (Bulimia Nervosa), using the psychophysiological assessment of some parameters, with an experimental design. The statistical analysis was performed using between-within parametric statistics when data were normally distributed and non-parametric when they were not. During the psychophysiological registration a significant difference in SCL and HR in baseline values was noticed. In particular AN subgroup showed SCL values definitely lower than BN subgroup and a steady profile besides a hypoactivation in HR. A significant increase in SCL parameter was obtained in AN subgroup in the long term evaluation, with a normalization of the values with $\mathrm{BN}$ subgroup. Improvement observed in SCL parameter in AN might be understood in light of new prospective theories that investigate the psychopathological characteristics associated with a given diagnostic category: in particular, a tendency for females suffering from EDs to "exhibit" intense emotional reactions on the behavioral and verbal levels, despite experiencing a small physiological activation. The greater physiological reactivity following the IT may reflect patients' learning of new cognitive strategies for processing and managing emotional experiences. Therefore psychophysiological assessment can be an important and objective help for a proper differential diagnosis and for treatment evaluation in a prospective of IT.
\end{abstract}

Keywords Psychophysiology, Assessment, Eating Disorders, Integrated Treatment Evaluation

\section{Introduction}

Although use of an IT approach for various psychopathologies is gradually spreading in clinical settings, current empirical research still tends to focus attention exclusively on the effectiveness of a single psychoactive element in the treatment of a specific clinical population, or to focus on the comparison of the observable effects following the psychotherapeutic treatment of different theoretical and methodological matrices $[1,2]$. Research on the therapeutic effectiveness of various interventions also raises a series of methodological problems related both to the choice of instruments used to assess a symptom's course and to different operational definitions of clinical improvement. The present study therefore attempted to match an "outcome" research model aimed at analyzing the "final" results of a treatment, to a "process" research model aimed at investigating how the recovery process manifests itself over the time [3].

Literature data demonstrate an evident link between Eating Disorders and the autonomic system. In both AN and $\mathrm{BN}$ it has been shown a close relationship between an alteration in the autonomic control of Heart Rate Variability (HRV) and the sympathovagal activity. As a matter of fact $\mathrm{AN}$ and $\mathrm{BN}$ patients seem to show unbalanced sympathovagal control of HRV due to relative sympathetic failure, prevalent vagal activity and impaired sympathetic activation after tilting, independently from their actual body weight $[4 ; 5]$.

Moreover it seems that, especially in $\mathrm{BN}$, exposure to idiosyncratic stress led to significant cardiovascular reactivity and to a further increase after stress exposure, but not to recovery. In addition, stress exposure resulted in reduced electrodermal activity [6].

Finally, the observation of a great number of researches regarding the difficulty to generalize empirical results obtained in the experimental studies to the clinical reality has been to some extent acknowledged. The samples used in empirical studies are often unrepresentative of the situations that therapists find themselves confronted with in their active practices [3]. Hence, the sample considered in the present study does not constitute an "experimental" group as much as a true and proper "clinical" sample, as it is comprised of patients treated in an outpatient context and not selected "a priori" in relation to specific variables.

So, according to literature, we supposed that an autonomic imbalance, identified through the Psychophysiological Registration, could be an objective parameter for a proper 
differential diagnosis and a useful instrument for monitoring IT effectiveness.

The principal aim of this study is to test these hypotheses: a differential diagnosis could be made by means values coming from a Psychophysiological Registration that could be useful for differentiate either the effective difference between the two diagnostic subgroups (AN and $\mathrm{BN}$ ) and the progressive changes during and after the IT. Furtermore, the psychophysiological approach is a useful tool to better assess the effectiveness of an integrated therapy in a clinical population that:

(a) satisfies the current international criteria for Eating Disorder (ED) diagnoses;

(b)concurrently underwent both a cognitive-behavioural psychotherapeutic treatment, and a controlled psychopharmacological support treatment.

The therapeutic effects investigated are therefore attributable to an "integrated therapy" (IT). IT is the combination of two interventions, a psychological and a pharmacological one. In this study the attention was focused on the variations achieved by the sample, divided into two diagnostic subgroups Anorexia Nervosa (AN) and Bulimia Nervosa (BN). Among other assessment techniques the psychophysiological assessment of some parameters, specifically the Electromyography of the forehead muscle (EMG), Skin Conductance Level (SCL), Peripheral Temperature (PT), Heart Rate (HR) and Inter Beat Interval (IBI) has been employed.

\section{Materials and Methods}

\subsection{Sample}

19 women between the ages 15 and 43 -years-old $(M=25,6$; $\mathrm{SD}=7,7$ ), who were suffering from an ED not directly attributable to a specific medical condition or endogenous pathology were subsequently recruited. The total sample was divided into two groups depending on whether the patients' predominant symptoms adhered to the DSM-IV-TR [7] criteria for the diagnosis of Anorexia Nervosa or Bulimia Nervosa. This subdivision was specifically conducted through different assessment tools by a mental health practitioner, according to symptoms and psychopathological characteristics reported by patients at the time of their initial intake. Thus the subdivision omitted patients' possible successive migrations toward behavior characterizing other psychopathological sub-profiles (for example, the conversion of the patient with a diagnosis of Anorexia Restricted-type to that of Anorexia Purging-type or to Bulimia). For this purpose, any presence of evident underweight (BMI <17.5) and of amenorrhea has been considered particularly significant, making it possible to identify and differentiate the group of anorexic patients (AN, $n=8)$ and the group of bulimic patients $(B N, n=11)$. All of the individuals underwent a cognitive-behavioral psychotherapeutic treatment as well as a pharmacological regimen considered appropriate for each case on the basis of the prevalent symptomatology and on its duration. The integrated intervention lasted for no less than one year, but duration varied from patient to patient. Drugs administration and management were made by a psychiatrist; dosage was adapted to patients' needs with monthly controls. Pharmacological treatment decreased or was interrupted with progresses gained with psychotherapy. Overall, in the cases the patient was particularly impaired, the therapy was prolonged until approximately two years and more than 80 psychotherapy sessions after the end of the initial four-session evaluation phase.

\subsection{Materials and Procedure}

The Psychophysiological Profile (PPP) was carried out for two treatment phases: diagnostic phase and termination. The purposes of the PPP administration were to gather information on the possible presence and consistency of a psychophysiological impairment as well as to verify the presence of a typical activation pattern for each subgroup (AN and $\mathrm{BN}$ ) [8,2]. The PPP recording is structured according the following three phases: "rest" phase or baseline (phase b; 6 minutes long) in which each patient is instructed to close his/her eyes and to remain as still and relaxed as possible. "Stress" phase (phase s; 4 minutes long) in which the patient is asked to perform a mental task consisting of subtracting the number 13 from the number 1007 and continuing to subtract 13 from each successive result that is obtained (MAT). "Recovery" phase (phase $\mathbf{r} ; 6$ minutes long) in which the patient is instructed to relax again. Four physiological parameters are recorded during each phase: Skin Conductance Level (SCL), Heart Rate (HR) and Inter Beat Interval (IBI), Peripheral Temperature (PT), and Electromyography of the forehead muscles (EMG).

\subsection{Statistical Analysis}

From the obtained data, the following descriptive statistics were computed:

- the mean and the standard deviation of the all psychophysiological parameters recorded for each of the PPP's three phases (phase b, s, r) in the two different treatment phases (phase 1st, 2nd), for the total sample and for the two subgroups (AN, BN)

In order to identify possible significant differences in the psychopathological characteristics of the two subgroups (AN, $\mathrm{BN})$, and in the two phases, the following analyses were conducted:

- the Kolmogorov-Smirnov test was made on the AN and BN subgroups' values for the four physiological parameters for the three phases of the PPP recorded during each phase, in order to verify data normal distribution;

- in presence of normal variables, we tested data homoskedasticity applying $\mathrm{F}$ test and statistical evaluation was performed using a two way ANOVA between-within model [Patology (AN, BN) - PPP 
phase $(\mathbf{b}, \mathbf{s}, \mathbf{r})]$ in a mixed design for repeated measurements. Post-hoc multiple comparisons analysis among "between" data were performed applying Tukey-Kramer HSD Test.

- in absence of a normal distribution of the data, differences between disorders (AN - BN) were analyzed using the non-parametric Mann -Whitney U test. The Bonferroni's correction for multiple comparisons was applied.

\section{Results}

The descriptive analyses computed from sample's PPP values recorded during diagnostic phase (phase 1), showed in BN subgroup moderately high baseline values in muscle tension (EMG $>4 \mu \mathrm{V}$ ) and normative values in the other parameters (SCL,PT,HR). There was a consistent activation in the EMG parameter during the stress phase and a meager activation in the other parameters. In the last phase, there was a slight recovery in EMG, complete recovery in HR, absent recovery in PT and a continuous SCL activation (Tab.1).

Table 1. Mean and Standard Deviation of BN subgroup's PPP recorded at the $1^{\text {st }}$ phase

\begin{tabular}{|c|c|c|}
\hline & Mean & Std. Deviation \\
\hline EMG_b & 4,6325 & 1,34923 \\
\hline SCL_b & 3,5600 & 2,07986 \\
\hline PT_b & 31,1658 & 1,94404 \\
\hline HR_b & 72,2075 & 10,56428 \\
\hline EMG_s & 5,4283 & 1,85641 \\
\hline SCL_s & 3,9442 & 2,49843 \\
\hline PT_s & 30,7950 & 2,26387 \\
\hline HR_s & 74,1925 & 10,93118 \\
\hline EMG_r & 5,0300 & 2,14788 \\
\hline SCL_r & 4,1175 & 2,15661 \\
\hline PT_r & 30,8242 & 2,41304 \\
\hline HR_r & 71,8917 & 10,55537 \\
\hline
\end{tabular}

AN subgroup also manifest a high baseline EMG, while SCL was definitely low (SCL $<2 \mathrm{mS}$ ), PT showed normal values and HR was moderately low. During the stress phase there was a consistent EMG activation, while all the other parameters showed a meager or absent activation. In the last phase all parameters, but EMG, displayed absent recovery (Tab.2).

BN subgroup recorded at the treatment termination (phase 2) still showed moderately high values in EMG baseline, and standard values in the other parameters. In the stress phase there was a consistent activation in each parameter. In the last phase there was a complete recovery in EMG and HR, while SCL recovery wasn't fulfilled and PT was constant (Tab.3).

In AN subgroup, recorded at phase 2, EMG remained moderately high, and the other parameters increased reaching normal values. During the stress phase, there was a slight activation of PT and HR, a consistent activation of EMG and absent SCL activation. In the last phase, EMG and
HR showed a consistent recovery, while SCL still increased and PT remained stable (Tab.4).

Table 2. Mean and Standard Deviation of AN subgroup's PPP recorded at the $1^{\text {st }}$ phase

\begin{tabular}{|c|c|c|}
\hline & Mean & Std. Deviation \\
\hline EMG_b & 3,5629 & 1,34989 \\
\hline SCL_b & 1,6986 & 0,77572 \\
\hline PT_b & 30,3829 & 2,57580 \\
\hline HR_b & 61,1500 & 5,09076 \\
\hline EMG_s & 4,8843 & 1,94134 \\
\hline SCL_s & 1,9786 & 0,86962 \\
\hline PT_s & 30,3400 & 2,45424 \\
\hline HR_s & 63,5729 & 5,97527 \\
\hline EMG_r & 3,8057 & 1,52059 \\
\hline SCL_r & 2,0971 & 1,30528 \\
\hline PT_r & 30,2771 & 2,39485 \\
\hline HR_r & 63,1386 & 6,78184 \\
\hline
\end{tabular}

Table 3. Mean and Standard Deviation of BN subgroup's PPP recorded at the $2^{\text {nd }}$ phase

\begin{tabular}{|c|c|c|}
\hline & Mean & Std. Deviation \\
\hline EMG_b & 4,3583 & 1,35514 \\
\hline SCL_b & 4,6283 & 2,35620 \\
\hline PT_b & 32,5925 & 1,75092 \\
\hline HR_b & 75,3300 & 11,59281 \\
\hline EMG_s & 5,7600 & 1,47830 \\
\hline SCL_s & 6,2742 & 4,14615 \\
\hline PT_s & 32,2217 & 2,31969 \\
\hline HR_s & 76,0483 & 10,67804 \\
\hline EMG_r & 4,4225 & 1,36501 \\
\hline SCL_r & 5,0925 & 3,01147 \\
\hline PT_r & 32,2067 & 2,20857 \\
\hline HR_r & 74,0167 & 10,74873 \\
\hline
\end{tabular}

Table 4. Mean and Standard Deviation of AN subgroup's PPP recorded at the $2^{\text {nd }}$ phase

\begin{tabular}{|c|c|c|}
\hline & Mean & Std. Deviation \\
\hline EMG_b & 4,0143 & 1,63278 \\
\hline SCL_b & 4,3586 & 1,05181 \\
\hline PT_b & 32,0643 & 1,62836 \\
\hline HR_b & 65,0114 & 8,13081 \\
\hline EMG_s & 6,1814 & 3,65172 \\
\hline SCL_s & 4,4629 & 1,76525 \\
\hline PT_s & 31,8286 & 1,55854 \\
\hline HR_s & 68,3357 & 6,19385 \\
\hline EMG_r & 4,4471 & 1,76115 \\
\hline SCL_r & 5,1300 & 2,27340 \\
\hline PT_r & 31,8871 & 1,67038 \\
\hline HR_r & 65,9714 & 9,37492 \\
\hline
\end{tabular}

Statistically significant differences emerged between $\mathrm{AN}$ and $\mathrm{BN}$ groups in Heart Rate. In the diagnostic phase, AN group showed in baseline significant lower values than BN group (Two-Way between-within ANOVA, Tukey-Kramer HSD Test, p <0,05) (Fig.1).

Kolmogorov-Smirnov test showed a normal distribution for HR ( $\mathrm{P}>0,1)$, that also showed a homoskedastic profile ( $\mathrm{F}$ test, $\mathrm{P}<0,05$ ), allowing us to analyze it by Two-Way ANOVA with Unequal Replications. All the other variables didn't comply normal distribution (always $\mathrm{P}<0,05$ ), in these case non parametric statistic has been used. 


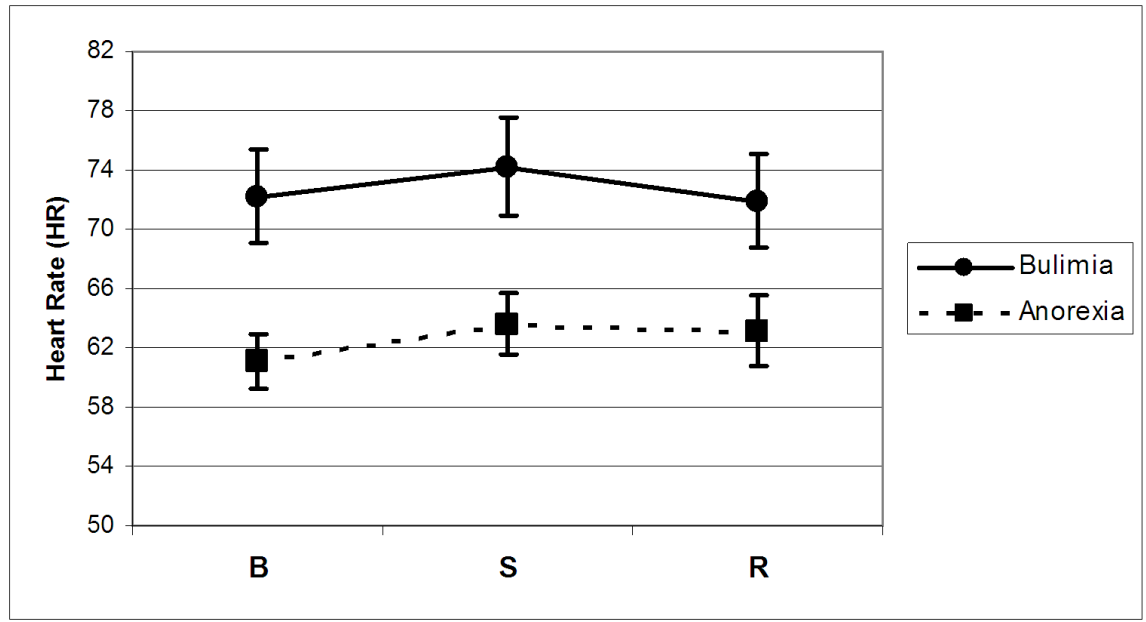

Figure 1. Mean \pm SE HR values recorded from bulimic and anorexic groups during the baseline (B), stress, (S), and recovery (R) at the diagnostic phase $\left(1^{\text {st }}\right)$

SCL parameters also showed statistically significant differences between two subgroups: the AN subgroup achieved values definitely lower than the $\mathrm{BN}$ subgroup (Mann-Whitney $\mathrm{U}$ test, $\mathrm{P}<0,01$ ) (Fig.2).

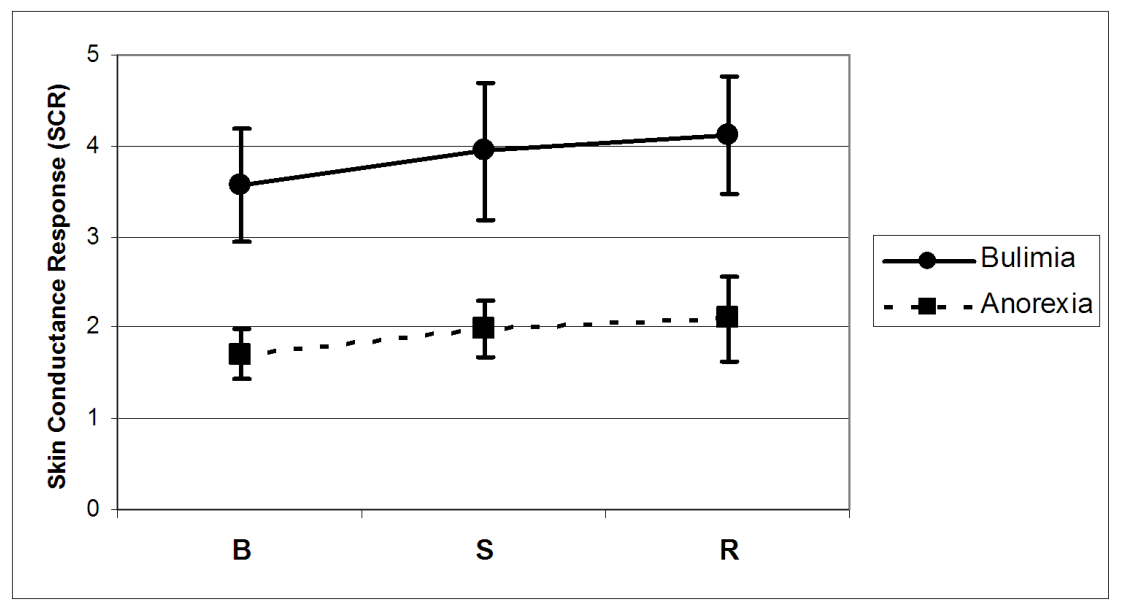

Figure 2. Mean \pm SE SCL values recorded from bulimic and anorexic groups during the baseline (B), stress, (S), and recovery (R) at the diagnostic phase $\left(1^{\text {st }}\right)$

Furthermore, looking at the long term evaluation (phase 2) a statistically significant difference emerged in SCL parameter. A significant increase was obtained in the subgroup with AN in the long term evaluation, with a normalization of the values with the BN subgroup (Fig.3). No statistical differences between two groups have been found in both EMG and PT recordings.

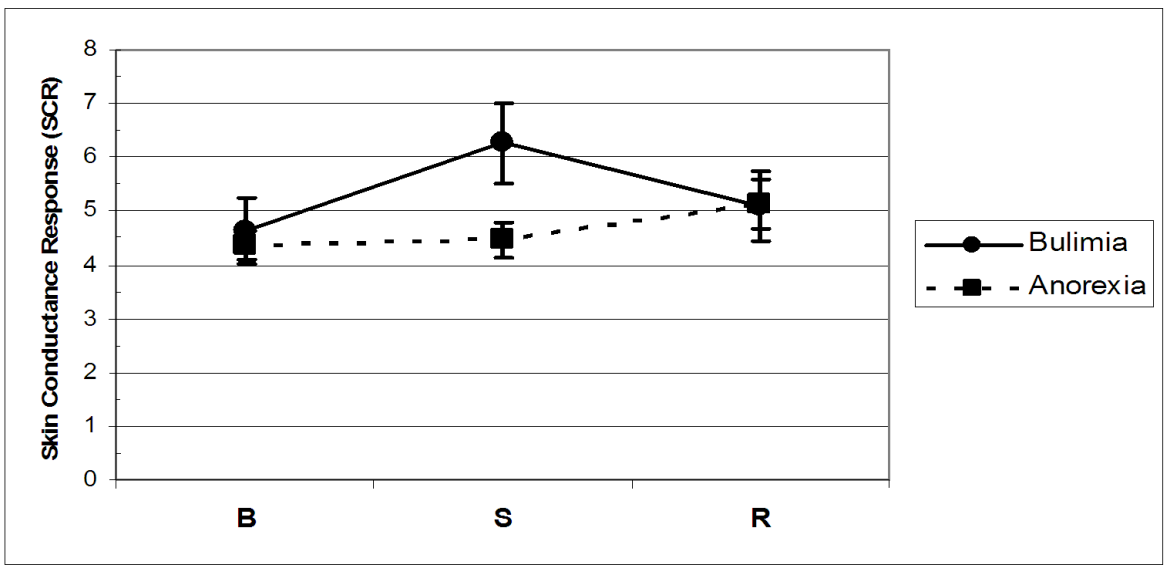

Figure 3. Mean \pm SE SCL values recorded from bulimic and anorexic groups during the baseline (B), stress, (S), and recovery (R) at the post treatment phase $\left(2^{\text {nd }}\right)$. 


\section{Conclusions}

From data analysis, some interesting characteristics emerged in two specific parameters: SCL and HR. The anorexic subgroup showed a significant lower activation in SCL parameter, and a meager SCL reactivity has frequently been observed in patients suffering from anorexia [2]. The innovative data deriving from this research, is the improvement, after treatment, of SCL level in anorexic patients, which showed a normalization trend towards the same values showed by the bulimic subgroup. Also the HR parameter showed a typical trend: at the diagnostic phase anorexic and bulimic groups' values were significantly different, in fact anorexic patients showed hypoactivation in this parameter. No significant HR improvements have been found after treatment, but the high HR variability [9] and the paucity of the sample could justify the absence of this outcome.

Improvement observed at the physiological level might be understood in light of new prospective theories that investigate the psychopathological characteristics associated with a given diagnostic category. Some experimental research demonstrates that individuals suffering from EDs tend to "exhibit" intense emotional reactions on the behavioral and verbal levels, despite experiencing a small physiological activation. Studies concerning gender differences in the expression of their emotional experiences have also shown that males and females do not differ as much in emotionality, as in learned response style, with women showing a greater tendency toward "externalization" of their emotions [10]. Thus, the greater physiological reactivity found in this study following the therapeutic intervention may be at least partially interpreted as reflecting the patients' learning of new cognitive strategies for processing and managing their emotional experiences. However, given the small sample examined, it is necessary to stress the usefulness of undertaking further investigation. In addition, monitoring the effects of any form of psychotherapy raises a number of problems relative to the measurement criteria, to decisions regarding when to carry out assessments, and to possible generalization of the obtained results. It is by now evident that the effects of treatment often do not involve only variables explicitly considered by the clinician, but much more global issues affecting the individual [11,12]. While testing the effectiveness of a treatment, considering differences between the values collected at different times or assessing the mean change obtained from patients belonging to the same diagnostic category seems to be a really useful tool. However must be considered that each individual's clinical profile can still hide its own idiosyncratic maintenance factors behind these nosological definitions. These idiosyncrasies are explained by the specific patient history, the relationship between the patient and his/her "environment," and the meanings that the patient cognitively attaches to events. Therefore, it becomes crucial to reconcile and integrate a more normative vision of the results with a more subjective vision that focuses on the peculiarities of the individual.

This research, according to what has been already underlined in literature, showed that, in the diagnostic phase, PPP can be an important tool in order to collect "objective" data, that can help clinicians formulating a proper differential diagnosis, specially referring to EDs, that frequently configure themselves as a "spectrum", with peculiar fluctuation from a psychopathological form to the other. Furthermore, PPP seems to be useful also to evaluate the treatment effectiveness: it provides information about the physiological recovery, and according to the concept of "response desynchronization", eventual improvements don't occur simultaneously in each "channel" of response (cognitive, behavioral, and physiological). Indeed, the physiologic and behavioural reaction to stress has been recognized as a liability factor for many organic and psychological disorders. Its earlier identification, makes possible to curb the negative effects deriving from psycho-social stress [13]. A physiological evaluation, can allow to the clinician to detect a real improvement before the patient's realization. It is surely undisputed that the clinician has to continue and monitor treatment until there is not only absence of symptoms, but also an objective assurance that the therapeutic changes have stabilized.

Ultimately, the use of a psychophysiological Registration as the PPP, has to be set in a perspective of "multidimensional approach", collecting data deriving from different channels, in order to have a various and complex patient's picture, leading to a proper diagnosis and to a correct intervention planning, properly tailed on the patient.

\section{REFERENCES}

[1] Gabbard, G. O., Kay, J. The fate of integrated treatment: whatever happened to the biopsychosocial psychiatrist?. American Journal of Psychiatry, Vol.158, No.12, 1956-1963, 2001.

[2] Pruneti, C., Giusti, M., Boem, A., Luisi M. Behavioral, psychophysiological and salivary cortisol modifications after short-term alprazolam treatment in patients with recent myocardial infarction. Italian Heart Journal, Vol.3, No.1, 53-59, 2002.

[3] Smeraldi, E., Cavallaro, R., Migone, P. Valutazione dei risultati terapeutici. In P. Pancheri, G. Cassano. Trattato italiano di psichiatria, Masson Editore, Milano, 1999.

[4] Yoshida, N. M., Yoshiuchi, K., Kumano, H., Sasaki, T., Kuboki, T. Changes in heart rate with refeeding in anorexia nervosa: a pilot study. Journal of psychosomatic research, Vol.61, No. 4, 571-575, 2006

[5] Murialdo, G., Casu, M., Falchero, M., Brugnolo, A., Patrone, V., Cerro, P. F., Ameri, P., Andraghetti, G., Briatore, L., Copello, F., Cordera, R., Rodriguez, G., Ferro, A. M. Alterations in the autonomic control of heart rate variability in patients with anorexia or bulimia nervosa: correlations between sympathovagal activity, clinical features, and leptin levels. Journal of Endocrinological Investigation. Vol.30. 
No.5, 356-362, 2007.

[6] Hilbert, A., Vögele, C., Tuschen-Caffier, B., Hartmann, A. S. Psychophysiological responses to idiosyncratic stress in bulimia nervosa and binge eating disorder. Physiology \& behavior, Vol.104, No.5, 770-777, 2011.

[7] American Psychiatric Association. Diagnostic and statistical manual of mental disorders, text revision (DSM-IV-TR). American Psychiatric Association, 2000

[8] G. D. Fuller. Biofeedback methods and procedures in clinical practice, Biofeedback Press, San Francisco, 1979

[9] Mazurak, N., Stein, J., Kipphan, S., Muth, E. R., Teufel, M., Zipfel, S., Enck, P. Heart rate variability in anorexia nervosa and the irritable bowel syndrome. Neurogastroenterology \& Motility, Vol.23, No.11, 470-478, 2011.
[10] Codispoti, O., Golfarini, G. Emozioni al femminile e disturbi del comportamento alimentare. In O. Codispoti, A. Simonelli. Narrazione ed attaccamento nelle patologie alimentari, Raffaello Cortina Editore, Milano, 2006.

[11] Parloff, M. B. Psychotherapy outcome research. In R. Michels, J. O. Cavenar, Psychiatry, Lippincott, Philadelphia, 1985.

[12] Pruneti, C., Lento, R. M., Fante, C., Carrozzo, E., Fontana, F. Autonomic arousal and differential diagnosis in clinical psychology and psychopathology. Italian Journal of Psychopathology, Vol.16, 43-52, 2010.

[13] Pruneti, C. The "P Stress Questionnaire": A new tool for the evaluation of stress-related behaviors. European Journal of Clinical Psychology and Psychiatry, Vol.6, 1-37, 2011. 